\title{
Schallemissionsanalyse beschichteter kohlenstofffaserverstärkter Kunststoffe
}

\author{
M. G. R. Sause und S. Horn \\ Universität Augsburg, D-86135 Augsburg
}

\section{$1 \quad$ Einleitung}

Zur Verbesserung der funktionalen Oberflächeneigenschaften von Kohlenstofffaserverbundwerkstoffen (CFK) werden zunehmend Beschichtungen eingesetzt. Diese können als Verschleißschutz oder zur Erhöhung der Leitfähigkeit [1] dienen. In der vorliegenden Untersuchung wurden galvanische Beschichtungen auf CFK-Substraten untersucht, die als Diffusionsbarriere für Leichtbautanksysteme zur Wasserstoffspeicherung dienen sollen. Die Schichtsysteme für diese Tankstrukturen wurden im Rahmen des von der EU geförderten Projektes „StorHy“ entwickelt und auf ihre Wasserstoffpermeabilität untersucht [2]. Eine grundlegende Problematik besteht dabei in der Definition und Beurteilung der mechanischen Belastbarkeitsgrenze, deren Überschreiten zu einem funktionalen Versagen derartiger Schichtsysteme führt. Im Falle von Diffusionsbarrieren stellen bereits kleinste Risse und Delaminationen schwerwiegende Versagensmechanismen dar, die zu einer deutlichen Abnahme der Barrierewirkung führen können [2] und somit diese funktionale Belastbarkeitsgrenze definieren. Mit Schältests oder Stirnzugversuchen lässt sich die Adhäsionskraft zwischen Beschichtung und Substrat gut bestimmen. Sie ist aber nicht hinreichend zur Festlegung dieser Belastbarkeitsgrenze der Beschichtung, da die mechanische Festigkeit einer Beschichtung oftmals weit vor dem Adhäsionsbruch erreicht wird. Die mechanische Festigkeit lässt sich wiederum gut in Einzelzugversuchen an den Beschichtungen ohne Substrat ermitteln. Auf Grund zusätzlicher Verspannungen zwischen Substrat und Beschichtung kann die funktionale Belastbarkeitsgrenze (Risse, Delamination) in Schichtsystemen jedoch unterhalb der mechanischen Festigkeit der Beschichtung liegen. Die Schallemissionsanalyse (SEA) bietet die Möglichkeit, mikroskopische Materialverformungen, wie beispielsweise Risse und Delaminationen, während einer Belastung der Probe im realitätsnahen Spannungsfall $\mathrm{zu}$ detektieren. Mittels Lokalisierungsverfahren, Mustererkennung und Frequenzanalysen kann zusätzlich deren Herkunftsort bestimmt und der zugrunde liegende Schadensmechanismus identifiziert werden [3].

\section{Messmethoden}

In der vorliegenden Untersuchung wurden für fünf verschiedene Beschichtungszusammensetzungen jeweils fünf Proben bei einer Belastung in 4-Punkt-Biegung nach DIN-EN-ISO 14125 untersucht, was einem realitätsnahen Spannungszustand für Beschichtungen in druckbeaufschlagten Tanksystemen nahe kommt. Das CFK-Substrat besteht aus sechs Gewebelagen mit $0^{\circ}-90^{\circ}$ Orientierung des Prepregmaterials SIGRATEX KDK 8054/120 und dem Matrixsystem Araldite LY 564 / HY 2954 mit den äußeren Abmessungen 100 mm x 15 mm x 2 mm. Auf diesem CFKSubstrat wurde mittig eine Beschichtung mit einer Abmessung von 25 mm x 15 mm aufgebracht. Als erste Lage wurde mittels elektrochemischer Abscheidung eine $10 \mu \mathrm{m}$ dicke Startschicht aus Nickel aufgebracht. Anschließend wurde mit einem galvanischen Verfahren die eigentliche funktionale Kupferbeschichtung mit einer Dicke von $10 \mu \mathrm{m}, 30 \mu \mathrm{m}$, bzw. $50 \mu \mathrm{m}$ aufgetragen. Zusätzlich wurde ein weiteres Schichtsystem mit einer $10 \mu \mathrm{m}$ dicken Startschicht aus Kupfer und 
anschließender funktionaler Kupferschicht von $50 \mu \mathrm{m}$ Dicke hergestellt. Diese Proben werden im Folgenden als NiCu10, NiCu30 und NiCu50, bzw. CuCu50-Proben bezeichnet. Eine schematische Darstellung des 4-Punkt-Biegeversuchs, sowie eine schematische Darstellung der Schichtsysteme ist in Bild 1 gezeigt.

Die bei Belastung auftretende Schallemission wurde mit zwei Breitbandsensoren (Typ: WD, Hersteller: PAC) in linearer Anordnung detektiert. Die Signale wurden dabei über einen $40 \mathrm{~dB}$ Vorverstärker und einen Bandpass im Frequenzbereich von $20 \mathrm{kHz}-1 \mathrm{MHz}$ geführt und mit einer Abtastfrequenz von $10 \mathrm{MHz}$ aufgezeichnet. Die Ankopplung der Sensoren an die Probe wurde mit einer mittelviskosen Silikonpaste und zwei Klammern realisiert.

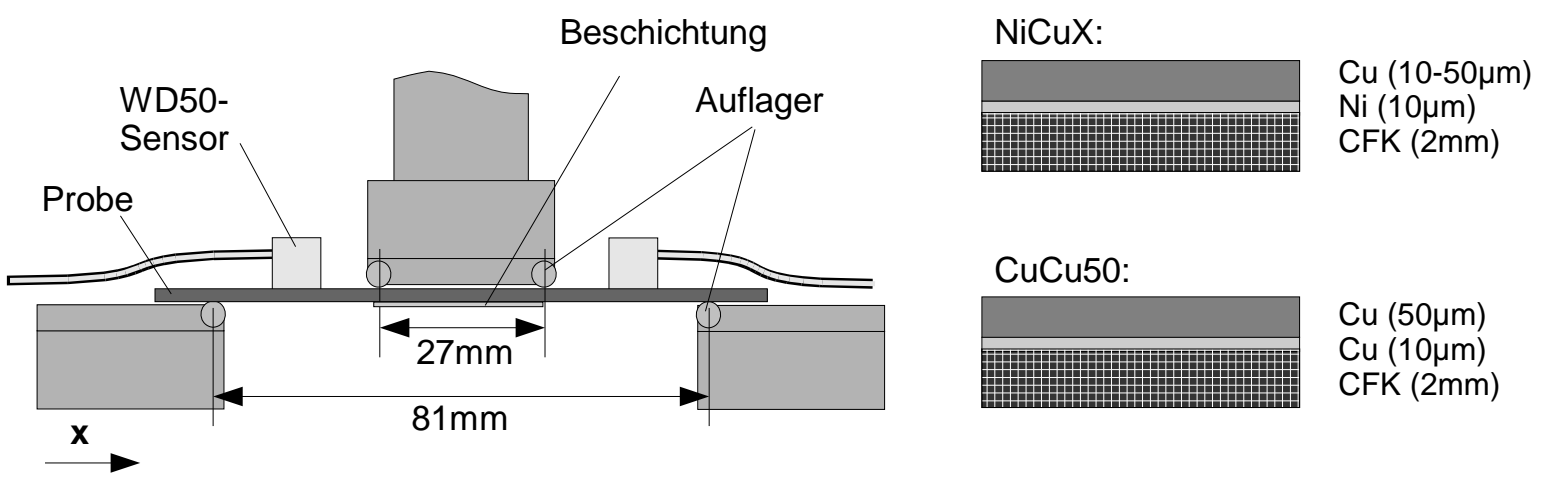

Bild 1: Schematische Darstellung des Versuchsaufbaus (links) und schematische Darstellung der beiden Schichtsysteme (rechts).

\subsection{Mikroskopische Analyseverfahren}

Zur vergleichenden Analyse der bei Bauteilbelastung entstandenen Schädigung wurden elektronenmikroskopische und ultraschallmikroskopische Aufnahmen der Proben durchgeführt. In Bild 2a und $2 \mathrm{~b}$ sind hierzu typische Bilder von NiCu-Proben nach einer Probenbelastung von $\varepsilon=0,8 \%$ Dehnung gezeigt. In allen NiCu-Proben wurden in den Ni-Schichten stark verzweigte Rissgeflechte beobachtet (s. Bild 2a), die eine unterschiedlich starke Ausprägung in Abhängigkeit der $\mathrm{Cu}$-Schichtdicke besitzen. Dieses Risswachstum findet nahezu unabhängig von weiteren Schädigungen der Cu-Schichten statt und führt insbesondere nicht zu einer großflächigen Ablösung der Ni-Schichten vom CFK-Substrat. In den $\mathrm{Cu}-S c h i c h t e n$ der NiCu-Proben wurden einzelne, kontinuierlich verlaufende Risse mit bevorzugter Ausrichtung orthogonal zur Spannungsrichtung (x-Richtung) gefunden (s. Bild 2b). Diese Risse nehmen mit steigender Belastung in Anzahl und Länge deutlich zu. Zusätzlich zu den Schädigungen der Beschichtungen durch Risse kommt es bei den NiCu-Beschichtungen abhängig von der $\mathrm{Cu}$-Schichtdicke zu teilweise stark ausgeprägten Delaminationen zwischen Ni-Startschicht und Cu-Schicht.
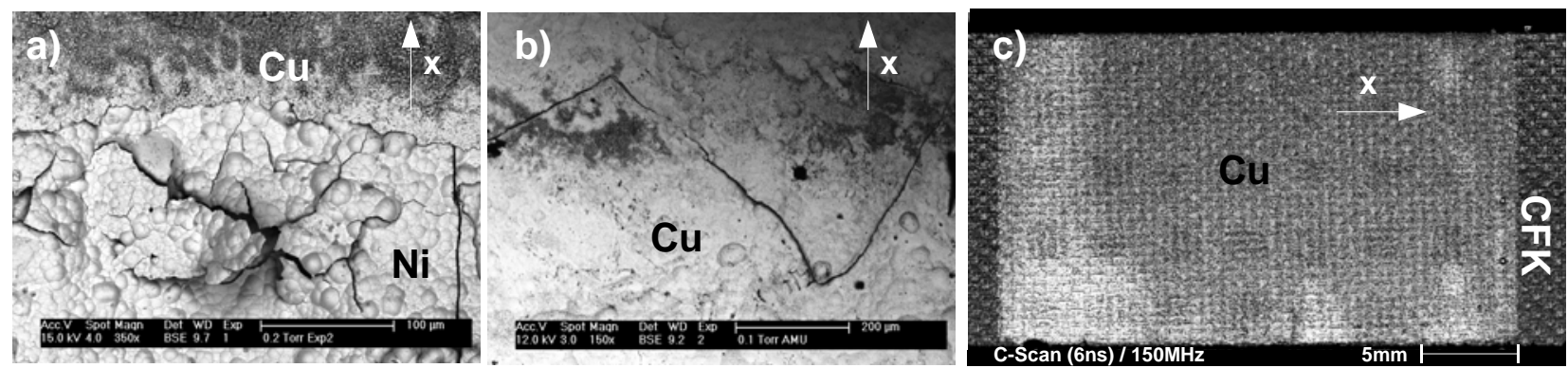

Bild 2: Elektronenmikroskopische $(\mathrm{a}+\mathrm{b})$ und ultraschallmikroskopische (c) Aufnahmen der Ni-Cu Beschichtungen in Aufsicht nach einer Belastung von $\varepsilon=0,8 \%$. 
Die delaminierte Fläche konnte hierbei mit ultraschallmikroskopischen Verfahren visualisiert und quantifiziert werden (s. Bild 2c). Die abgelösten Stellen weisen in der Intensitätsdarstellung durch veränderte Schichtspannungszustände, hervorgerufen durch fehlende Anhaftung, deutlich höhere Beiträge (weiße Färbung) auf. In Abhängigkeit von der Probendehnung wurde beobachtet, dass die Delamination zunächst an den Rändern der Beschichtung beginnt und bei zunehmender Belastung in Richtung der Probenmitte fortschreitet. Für die CuCu-Proben wurden hingegen erst bei hohen Belastungen $(\varepsilon>0,7 \%)$ Oberflächenrisse analog zu denen der NiCu-Proben beobachtet. Eine Ablösung der Beschichtung vom Substrat oder Delamination zwischen den beiden Cu-Schichten wurde hier nicht beobachtet.

\subsection{Schallemissionsanalyse}

Die während der Probenbelastung aufgezeichneten Schallemissionssignale wurden zunächst mit einem linearen Ortungsverfahren innerhalb eines Bereiches von $40 \mathrm{~mm}$ um die Probenmitte lokalisiert. In Bild 3a ist die Anzahl der lokalisierten Signale in Abhängigkeit der Dehnung für das CFK-Substrat ohne Beschichtung, eine NiCu10-, eine NiCu30-, eine NiCu50- und eine CuCu50Probe dargestellt. Es zeigt sich, dass der Einsatz der Schallemission in beschichteten Proben deutlich früher stattfindet als in reinem CFK. Da in keiner unbeschichteten CFK-Probe für $\varepsilon<0,8 \%$ Schallemission detektiert wurde, ist anzunehmen, dass alle Signale in beschichteten Proben unterhalb dieser Schwelle aus Schädigungen in der Beschichtung stammen. Zur Untersuchung der Korrelation zwischen typischen Schallemissionssignalen und den drei mikroskopisch beobachteten Schädigungsarten (Ni-Risse, Cu-Risse und Delamination zwischen Ni- und Cu-Schicht) wurden die aufgezeichneten Wellenformen für $\varepsilon<0,8 \%$ Dehnung mit einem Mustererkennungsverfahren nach Anastassopoulos et. al. untersucht [4].

Hierzu wurde in dem verwendeten Softwarepacket Noesis aus den Wellenformen der Schallemissionssignale zunächst Wellenformparameter extrahiert. Anschließend werden die Wellenformparameter normalisiert und in ihren Eigenwertraum projiziert. Innerhalb dieser Projektion wird nun nach Häufungspunkten mittels Mustererkennungsalgorithmen gesucht. Die Güte der erreichten Partitionierung der Wellenformen lässt sich durch statistische Parameter beschreiben. Eine hohe Güte der Partitionierung lässt dabei auf eine sinnvolle Unterteilung der Schallemissionssignale in verschiedene Klassen schließen. Die Anzahl der Partitionen deutet auf die wahrscheinlichste Anzahl von Schädigungsarten hin. Hierzu werden die Parameter $R_{i j}$ und $\tau$ eines Datensatzes, wie in Bild 3b beispielhaft für eine NiCu30-Probe gezeigt, in Abhängigkeit der Klassenanzahl untersucht:

$$
\begin{aligned}
& \mathrm{R}_{\mathrm{ij}}=\frac{\mathrm{D}_{\mathrm{i}}+\mathrm{D}_{\mathrm{j}}}{\mathrm{D}_{\mathrm{ij}}} ; \\
& \tau_{\mathrm{ij}}=\frac{\min \left(\mathrm{D}_{\mathrm{ij}}\right)}{\max \left(\mathrm{D}_{\mathrm{k}}\right)} ;
\end{aligned}
$$

Dabei werden mit $D_{i}$ und $D_{j}$ die mittleren Abstände aller Klassenelemente und mit $D_{i j}$ der Abstand der Klassenmittelpunkte bezeichnet, $\min \left(\mathrm{D}_{\mathrm{ij}}\right)$ stellt den minimalen Abstand zweier Klassen und $\max \left(D_{k}\right)$ den maximalen Abstand zweier Klassenelemente dar. Folglich unterscheiden sich die Klassen stärker voneinander, wenn niedrige Werte für $\mathrm{R}$ und hohe Werte für $\tau$ erreicht werden.

Für jede gefundene Klasse wurde zusätzlich das gemittelte Frequenzspektrum mit einer selbst entwickelten Software errechnet. Durch das Verhältnis der Frequenzanteile bei hohen und niedrigen Frequenzen kann die vorwiegende Abstrahlrichtung der Schallemissionsquelle ermittelt werden, falls frequenzabhängige Dämpfung und Dispersion bei der Laufzeit des Signals vernachlässigbar sind [5]. Dies ist bei den vorliegenden Probenabmessungen mit einem maximalen Abstand 
zwischen Schallemissionsquelle und Sensor von $40 \mathrm{~mm}$ der Fall. Mit dieser Methode lässt sich insbesondere zwischen Rissen (Abstrahlrichtung in der Probenebene) und Delamination (Abstrahlrichtung orthogonal zur Probenebene) unterscheiden, da diese zu unterschiedlichen Frequenzbeiträgen bei Frequenzen oberhalb von $400 \mathrm{kHz}$ führen.
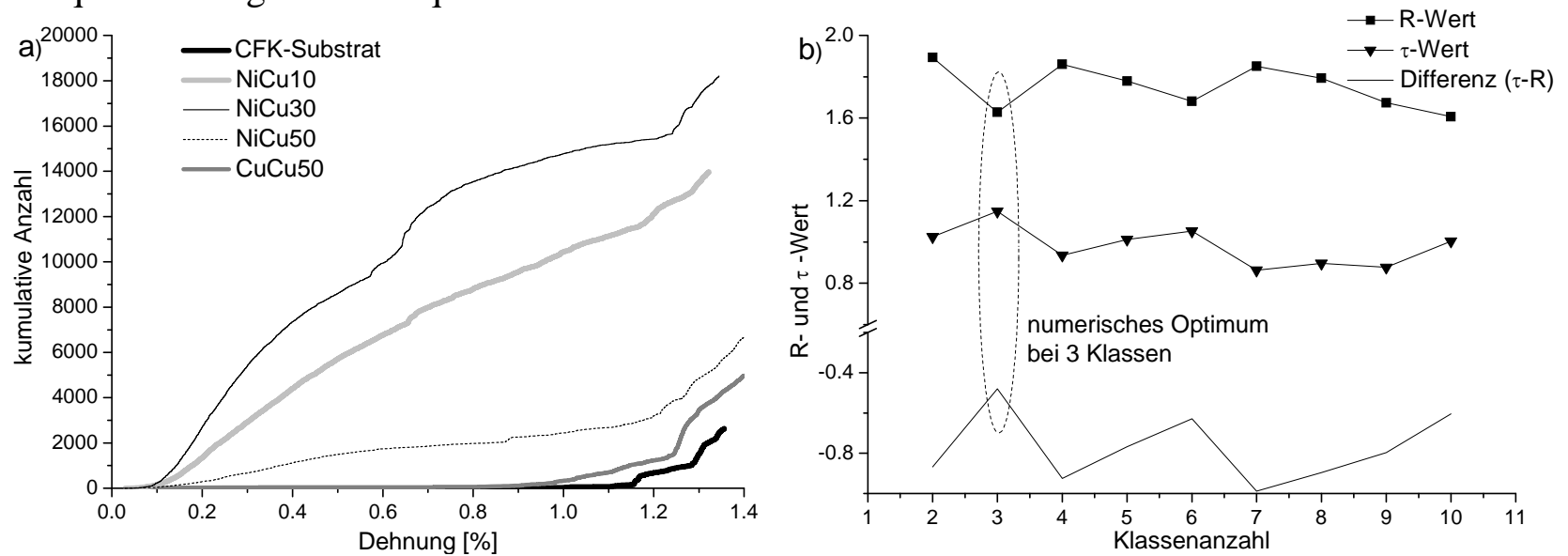

Bild 3: Dehnungsabhängige Anzahl der Schallemissionssignale verschiedener Proben (a) und Untersuchung der Abhängigkeit von $\mathrm{R}$ und $\tau$ von der Klassenanzahl einer NiCu30-Probe (b).

\section{Untersuchungsergebnisse}

\subsection{Ni-Cu Beschichtungen}

Für die reine Ni-Beschichtung konnte bei der Untersuchung der Parameter $\mathrm{R}$ und $\tau$ in Abhängigkeit der Klassenanzahl kein eindeutiges numerisches Optimum gefunden werden. Dies ist konsistent mit den mikroskopischen Beobachtungen, die auf nur eine Schädigungsart (Ni-Risse) hindeuten. Die untersuchten Proben zeigen dabei untereinander eine gute Übereinstimmung der Schallemissionssignale in Lokalisierung (Dichteverteilung entlang der x-Achse), dehnungsabhängiger Anzahl und mittlerem Frequenzspektrum.

Im Unterschied dazu wurden bei NiCu10-Proben numerische Optima der R- und $\tau$-Werte bei zwei, bzw. drei Klassen gefunden. Dieser Unterschied deutet auf eine probenspezifisch unterschiedliche Entwicklung der Schädigung hin. In Proben mit nur zwei Klassen wurde mikroskopisch neben dem Auftreten von Ni-Rissen nur großflächige Delamination zwischen der Ni- und der Cu-Schicht beobachtet, bei den NiCu10 Proben mit drei Klassen zusätzlich die Entstehung von Cu-Rissen. Die Abwesenheit von Rissen in der Cu-Schicht wird auf die niedrige Haftfestigkeit zwischen Ni- und Cu-Schicht zurückgeführt, die einen Spannungsabbau durch Rissbildung in der Cu-Schicht verhindert. In allen NiCu10-Proben wurde zudem eine Klasse gefunden, die im Lokalisierungsbild, der dehnungsabhängigen Entwicklung ihrer Anzahl und in ihrem gemittelten Frequenzspektrum mit den Schallemissionssignalen der reinen Ni-Beschichtungen übereinstimmt. Die in Bild 4a dargestellten drei Frequenzspektren einer NiCu10-Probe weisen zudem charakteristische Unterschiede auf. Dies lässt auf Grund der Intensitäten oberhalb von $400 \mathrm{kHz}$ und dem Lokalisierungsbild eine Unterscheidung zwischen Cu-Rissen (einzelne, abgegrenzte Positionen) und Delamination (flächige Lokalisierung) zu.

Bei den NiCu30-Proben weisen die Parameter R und $\tau$ ein numerisches Optimum bei 3 bzw. 4 Klassen auf. Die zusätzlich auftretende vierte Klasse besteht allerdings ausschließlich aus mehrfach reflektierten Signalen, denen kein zusätzlicher Schädigungsmechanismus zu Grunde liegt [3]. Die verbleibenden drei Klassen lassen sich analog der Überlegungen zu den NiCu10-Proben in NiRisse, Cu-Risse und Delamination einteilen. Die entsprechenden gemittelten Frequenzspektren einer NiCu30-Probe sind in Bild 4b gezeigt und zeigen gute Übereinstimmung mit den bei Ni- und 
NiCu10-Proben gefundenen Spektren. Im Unterschied zu den NiCu10-Proben geht der Anteil der Wellenformen, die der Delamination zugeordnet werden zu Gunsten der Cu-Risse deutlich zurück. In Übereinstimmung damit wurde mittels Ultraschallmikroskopie ein deutlicher Rückgang der delaminierten Fläche bei NiCu30-Proben festgestellt. Dies ist auf die deutliche Zunahme der Haftfestigkeit zwischen Ni- und Cu-Schicht zurückzuführen, die in Schältests nach ASTM-Norm B533-85 zu 0,13 kN/m für NiCu10-Proben und 0,56 kN/m für NiCu30-Proben bestimmt wurde. Aufgrund dieser verbesserten Haftfestigkeit wechselt der dominante Schadensmechanismus von Delamination hin zu Rissbildung in der Cu-Schicht.
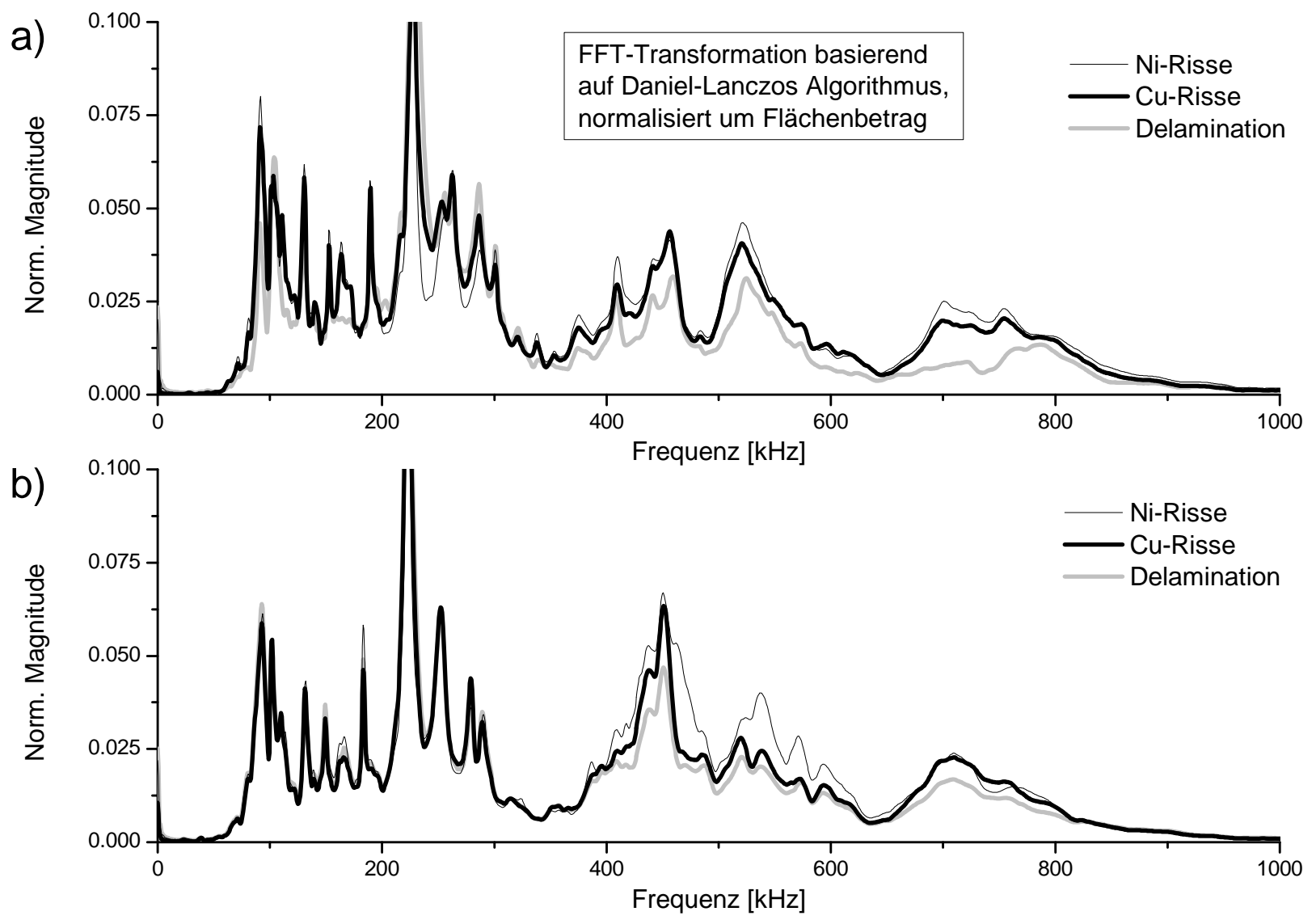

Bild 4: Gemitteltes Frequenzspektren der über Mustererkennung gefundenen Klassen einer NiCu10 (a) und einer NiCu30-Probe (b)

Für die NiCu50 Proben deuten die Parameter $\mathrm{R}$ und $\tau$ ebenfalls auf drei bzw. vier Klassen und entsprechende Schadensmechanismen hin. Eine ausführliche Diskussion hierzu ist in [3] zu finden. Im Unterschied $\mathrm{zu}$ den NiCu10 und NiCu30-Proben geht die Gesamtzahl der Schallemissionssignale jedoch deutlich zurück (s. Bild 3a), wobei die Gewichtung der Delamination erneut zu Gunsten der Zahl der Cu-Risse stark abnimmt. Mikroskopische Analysen zeigen konsistent hierzu einen deutlichen Rückgang der Schädigungen durch Delamination, Cu-Risse und Ni-Risse im Vergleich zu NiCu30-Proben. Diese höhere Belastbarkeit ist einerseits auf die erneute Zunahme der Haftfestigkeit auf über $1,20 \mathrm{kN} / \mathrm{m}$ und andererseits auf die durch die

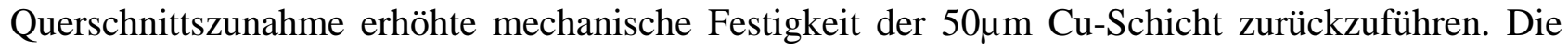
geringere Anzahl der Schallemissionssignale deutet grundsätzlich auf eine höhere Belastbarkeit der Beschichtung hin, da die Energiefreisetzungsrate der bruchmechanischen Energie proportional zur Energie der Schallemissionssignale ist [6]. 


\subsection{Cu-Cu Beschichtungen}

Ein Vergleich der dehnungsabhängigen Entwicklung der Schallemissionsenergie einer NiCu50 und einer CuCu50-Probe ist in Bild 5 gezeigt. Man erkennt bei der CuCu50 im Vergleich zu der NiCu50-Probe eine deutliche Verringerung der Anzahl der Schallemissionssignale unterhalb von $\varepsilon=0,7 \%$ bei gleichzeitig niedrigerem mittleren Energieniveau der aufgezeichneten Schallemissionssignale. Eine Untersuchung mit Mustererkennungsverfahren deutet darauf hin, dass die Signale der CuCu50 Probe bei niedrigen Dehnungen eine eigenständige Klasse bilden. Diese wird auf die Ablösung kugelförmiger Oberflächenpartikel unterhalb von $\varepsilon=0,7 \%$ zurückgeführt, die durch mikroskopische Analysen nachgewiesen wurden. Dies stimmt gut mit dem beobachteten sporadischen Auftreten und dem weit gestreuten Lokalisierungsbild der Schallemissionssignale überein. Schädigung in Form von Rissen oder Delamination wurden für $\varepsilon<0,7 \%$ nicht beobachtet.
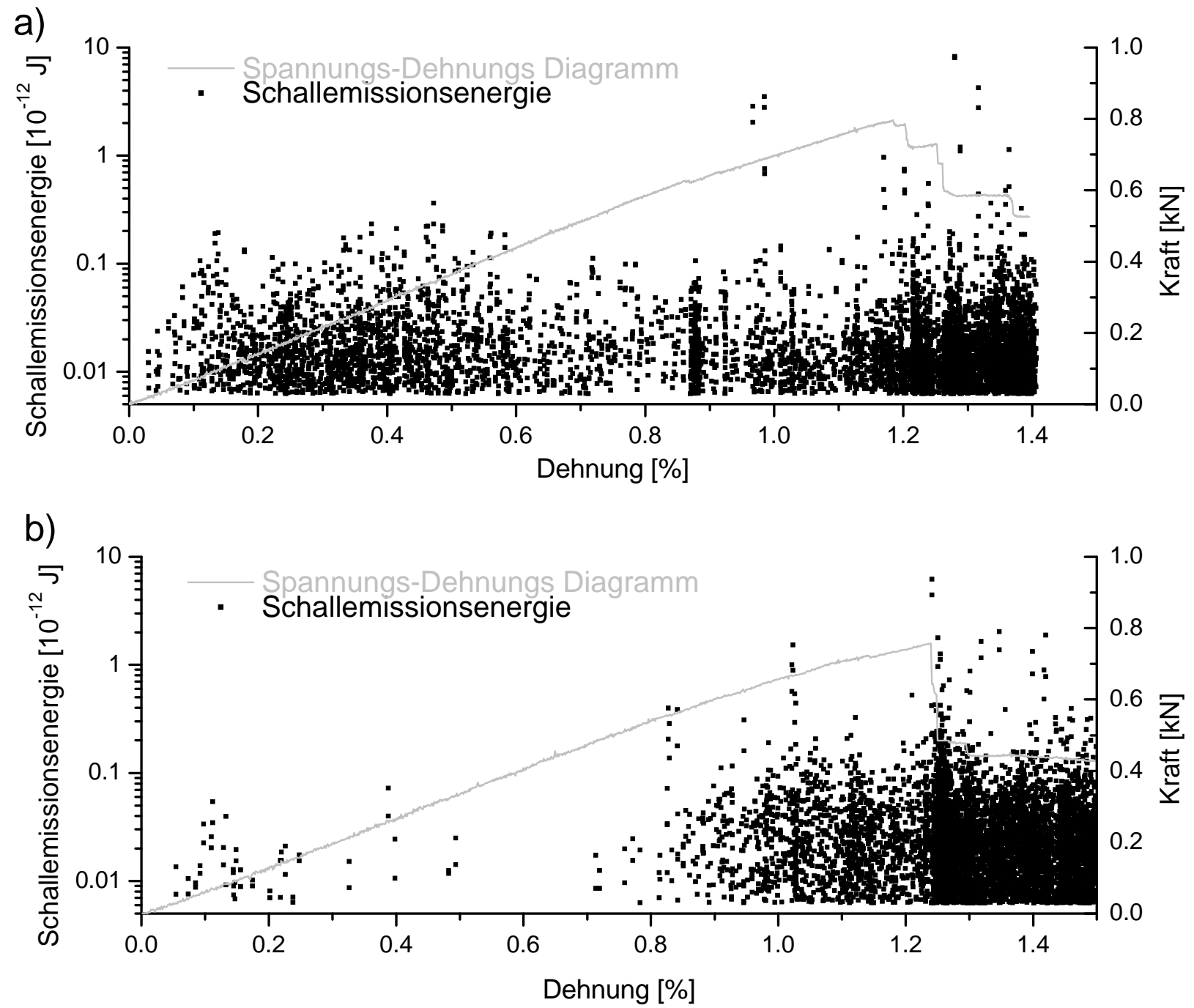

Bild 5: Dehnungsabhängige Entwicklung der Schallemissionsenergie einer NiCu50 (a) und einer CuCu50-Probe (b) zusammen mit den jeweiligen Spannungs-Dehnungs-Diagrammen

Damit liegt die funktionale Belastungsgrenze im CuCu50-Schichtsystem oberhalb von $\varepsilon=0,7 \%$. Diese deutlich überlegene mechanische Belastbarkeit ist auf die bessere Haftfestigkeit zwischen den beiden $\mathrm{Cu}$-Schichten im Vergleich zu den NiCu-Schichtsystemen zurückführen. Die erste chemisch aufgebrachte Lage weist in beiden untersuchten Schichtsystemen eine sehr starke Haftfestigkeit zum CFK-Substrat auf. In den Ni-Startschichten wurde allerdings eine Rissbildung bei geringeren 
Belastungen beobachtet als bei Cu-Startschichten. Die dadurch entstehende Spannung an der NiCu-Grenzfläche kann sich entweder durch vollständige oder teilweise Delamination der $\mathrm{Cu}-$ Decklage abbauen. Im letzteren Fall können lokale Spannungsmaxima auftreten, die eine vorzeitige Rissbildung in der Cu-Schicht zur Folge haben können.

\section{$4 \quad$ Zusammenfassung}

Es wurden galvanisch abgeschiedene Kupferschichten variierender Dicke mit Nickel oder Kupfer Startschicht auf CFK-Substraten mittels Schallemissionsanalyse unter mechanischer Belastung untersucht. Unter mechanischer Belastung treten bei unterschiedlicher Dehnung Risse in der NiStartschicht, Risse in der Cu-Schicht sowie Delamination zwischen Startschicht und Cu-Schicht auf. Die während der Belastung aufgezeichneten Schallemissionssignale konnten mittels Mustererkennungsverfahren in Klassen eingeteilt werden. Für alle Schichtsysteme konnte ein eindeutiger Zusammenhang zwischen den mikroskopisch nachgewiesenen unterschiedlichen Schädigungen und den durch das vorgestellte numerische Optimierungsverfahren erzielten Klassenanzahlen gefunden werden. Die zu jeder Klasse zugehörigen gemittelten Frequenzspektren weisen untereinander signifikante Unterschiede auf und stimmen in ihrem Lokalisierungsbild und ihrer Häufigkeit mit den assoziierten Schadensmechanismen (Risswachstum und Delamination) überein. Anhand der stark unterschiedlichen Ausprägung der Schallemissionsereignisse, sowie vergleichender mikroskopischer Analysen konnte gezeigt werden, dass die untersuchten $\mathrm{CuCu}-$ Beschichtungen den NiCu-Beschichtungen in ihrer funktionalen Belastbarkeitsgrenze deutlich überlegen sind.

Die durchgeführten Untersuchungen geben zu der Hoffnung Anlass, dass die Kombination von Mustererkennungsverfahren und Frequenzanalyse als sehr erfolgversprechend für die Schadensdiagnose von beschichteten Systemen oder für die Online-Strukturüberwachung von hybriden Bauteilen betrachtet werden kann.

\section{$5 \quad$ Literatur}

[1] R. W. Evans, NASA Contractor Report 4784 1997, 1-71

[2] D. Schultheiß, Dissertation 2007

[3] M. G. R. Sause, S. Horn, J. Acoustic Emission 2009, 24 (akzeptiert)

[4] A. A. Anastassopoulos, T. P. Philippidis, J. Acoustic Emission 1995, 13, 11-21

[5] J. Bohse, J. Acoustic Emission 2004, 22, 208-223

[6] J. Bohse, Compos. Sci. Tech. 2000, 60, 1213-1226 